\title{
DC link current simulation of voltage source inverter with random space vector pulse width modulation
}

\author{
Guoqiang Chen and Jianli Kang \\ School of Mechanical and Power Engineering, Henan Polytechnic University, Jiaozuo 454003, China
}

\begin{abstract}
Aiming at analysis complexity, a simulation model is built and presented to analyze and demonstrate the characteristics of the direct current (DC) link current of the three-phase two-level inverter with the random space vector pulse width modulation (SVPWM) strategy. The developing procedure and key subsystems of the simulation model are given in detail. Several experiments are done using the simulation model. The results verify the efficiency and convenience of the simulation model and show that the random SVPWM scheme, especially the random switching frequency scheme, can efficiently suppress the harmonic peaks of the DC link current.
\end{abstract}

\section{Introduction}

The voltage source inverter (VSI) has been widely in adjustable frequency drives (AFDs) in all kinds of fields [1]. For example, the drive system of the electric vehicle in the drive mode can be simplified in Figure 1. The battery provides the direct current (DC) power, the DC link transmits the DC voltage to the inverter, and the ride-through capacitors are connected to the DC link cables to store the harmonic energy. The inverter changes DC to alternating current (AC) to feed the load, the AC motor [2,3]. The inverter uses semiconductor switches to make the load terminal connect to the positive or negative rail of the DC link. The semiconductor switch includes the insulated gate bipolar transistor (IGBT), the insulated gate commutated thyristor (IGCT), and the injection enhanced gate transistor (IEGT), the gate turn-off thyristor (GTO), and so on. The three-phase two-level inverter shown in Figure 1 is mainly composed of 6 switches. The switches are always fully controllable and can be turned on and off by the third leads, the gate leads $\mathrm{T}_{1}, \mathrm{~T}_{2}, \mathrm{~T}_{3}, \mathrm{~T}_{4}, \mathrm{~T}_{5}$ and $\mathrm{T}_{6}$ in Figure 1.

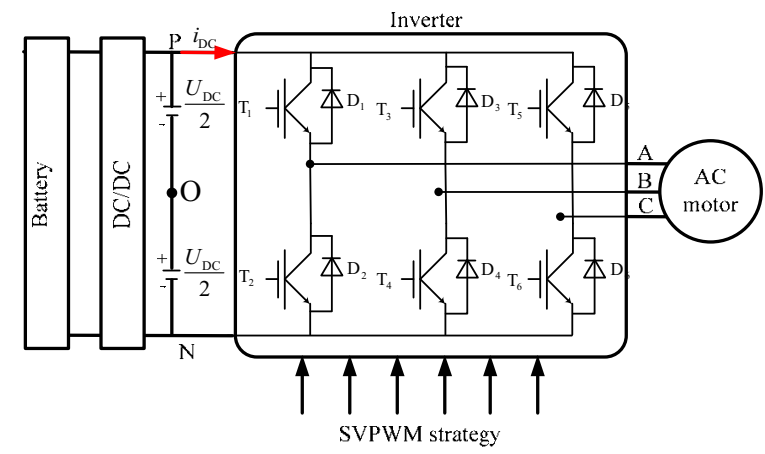

Figure 1. Drive system of electric vehicle in drive mode.
The six gate lead control signals are generated using the space vector pulse width modulation (SVPWM) strategy $[4,5]$. The SVPWM strategy uses the specific algorithm to compute the on and off state duration time of the six switches and generates the PWM gate control signals, so it is very convenient to implement in the digital control system. The input current $i_{\mathrm{DC}}$ of the inverter consists of high frequency rectangular stream of pulses with changeable widths and magnitudes [6]. The current pulses of the DC link current are phase current segments that flow through the DC link cable. The widths and magnitudes of the DC link current pulses highly depend on the AC motor speed and load torque, the SVPWM switching frequency and the SVPWM strategy. Besides the DC component, there are plenty of harmonics in the DC link current. The DC component that flows through the battery is corresponding to the output power of the AC motor. And the AC harmonics flow through the ride-through capacitor. The harmonic currents have significant influences on the working life of the capacitors and electromagnetic radiation [7]. The issues on how to compute the harmonic spectrum and the capacitor current RMS value of the DC link current and determine the capacitor volume have gained plenty of study. J.W. Kolar and S.D. Round [7] derived the analytical expression for the current stress on the DClink capacitor. T.D. Nguyen et al. [8] proposed a new PWM strategy called Uni-DCPWM that can effectively reduce the RMS current value of the DC link capacitor. N. Rouhana et al. [6] presented a method to analyze the DC-link current harmonic components for the Uni-DC PWM and the traditional SVPWM strategy using the double Fourier integral formulation. J. Hobraiche et al. [9] proposed an optimal strategy that uses selective harmonic elimination PWM to control the DC-link current harmonics. X. Pei et al. [10] discussed analysis and calculation of the DC link current and voltage

\footnotetext{
${ }^{a}$ Corresponding author: jz97cgq@sina.com
} 
ripples for a three-phase inverter with unbalanced load. The above study is focused on the deterministic SVPWM strategy. The volt-second balance principle that the SVPWM strategy adopts results in plenty of harmonics in the output waveforms of the inverter besides the required fundamental or first harmonic. The phenomenon is most serious in the deterministic SVPWM strategy because the harmonics around the multiples of the switching frequency have large magnitudes. The random strategy has been invented to suppress the peak harmonics and reduce the serious undesirable effects through spreading the harmonics continuously to a wideband area [11-19]. The DC link current presents tremendous difference in the random SVPWM strategy. The theoretical analysis and the analytical expression of the DC link current characteristic are always complicated and tedious. Therefore a simulation model is built and the characteristic of the DC link current with the random SVPWM strategy is discussed based on the simulation results in the paper.

\section{Principle of random SVPWM}

The topology of the inverter discussed in the paper is shown in Figure $1[2,4,5]$. The DC link voltage is $U_{\mathrm{DC}}$. The inverter has 8 permissible states that are corresponding to 8 basic space vectors as shown in Figure 2. An arbitrary command/reference voltage vector $\vec{U}_{\mathrm{s}}$ with the vector amplitude $U_{\mathrm{o}}$ and the phase angle $\theta$ inside the hexagon region shown in Fig. 2 can be generated by two adjacent active vectors (for example, $\vec{U}_{1}$ and $\vec{U}_{2}$ in the first sextant) and the zero vectors. The on-state duration time $T_{1}$, $T_{2}$ and $T_{0}$ of the three basic vectors are determined by the identical volt-second balance at the periodical time interval/switching period $T_{\mathrm{s}}$ using Equation (1). There are large numbers of vector operation modes that satisfy this equation.

$$
T_{\mathrm{s}} \vec{U}_{\mathrm{s}}=T_{1} \vec{U}_{1}+T_{2} \vec{U}_{2}
$$

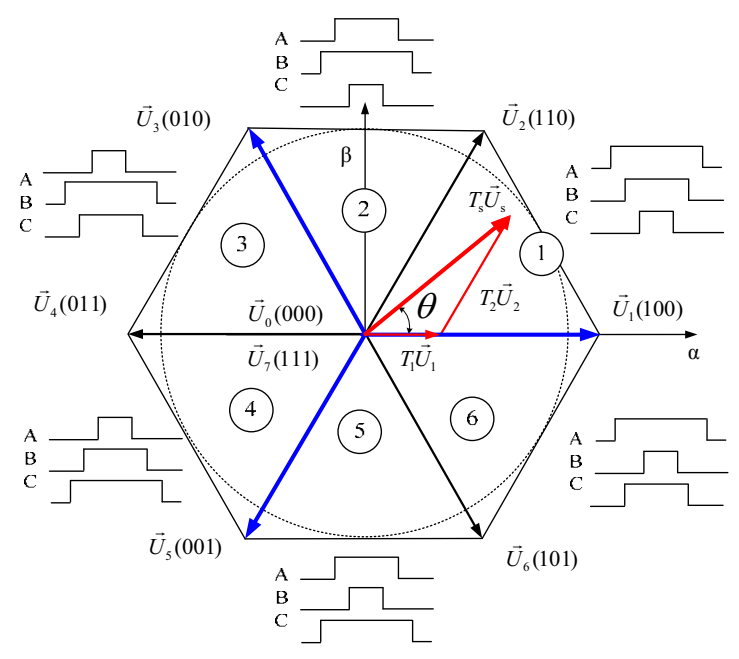

Figure 2. Vector diagram and vector summation method.
For the commonly used 7-segment SVPWM pattern operation of the inverter, the PWM waveforms in the 6 sextants are shown in Figure 2. The vector sequences in the 6 sextants are shown in Table 1 .

Table 1. Vector sequences of 7-segment SVPWM pattern.

\begin{tabular}{|c|l|}
\hline Sextant No. & \multicolumn{1}{|c|}{ Vector sequence } \\
\hline 1 & $\vec{U}_{0}(000) \rightarrow \vec{U}_{1}(100) \rightarrow \vec{U}_{2}(110) \rightarrow \vec{U}_{7}(111)$ \\
& $\rightarrow \vec{U}_{2}(110) \rightarrow \vec{U}_{1}(100) \rightarrow \vec{U}_{0}(000)$ \\
\hline 2 & $\vec{U}_{0}(000) \rightarrow \vec{U}_{3}(010) \rightarrow \vec{U}_{2}(110) \rightarrow \vec{U}_{7}(111)$ \\
& $\rightarrow \vec{U}_{2}(110) \rightarrow \vec{U}_{3}(010) \rightarrow \vec{U}_{0}(000)$ \\
\hline \multirow{2}{*}{3} & $\vec{U}_{0}(000) \rightarrow \vec{U}_{3}(010) \rightarrow \vec{U}_{4}(011) \rightarrow \vec{U}_{7}(111)$ \\
& $\rightarrow \vec{U}_{4}(011) \rightarrow \vec{U}_{3}(010) \rightarrow \vec{U}_{0}(000)$ \\
\hline & $\vec{U}_{0}(000) \rightarrow \vec{U}_{5}(001) \rightarrow \vec{U}_{4}(011) \rightarrow \vec{U}_{7}(111)$ \\
& $\rightarrow \vec{U}_{4}(011) \rightarrow \vec{U}_{5}(001) \rightarrow \vec{U}_{0}(000)$ \\
\hline 5 & $\vec{U}_{0}(000) \rightarrow \vec{U}_{5}(001) \rightarrow \vec{U}_{6}(101) \rightarrow \vec{U}_{7}(111)$ \\
& $\rightarrow \vec{U}_{6}(101) \rightarrow \vec{U}_{5}(001) \rightarrow \vec{U}_{0}(000)$ \\
\hline 6 & $\vec{U}_{0}(000) \rightarrow \vec{U}_{1}(100) \rightarrow \vec{U}_{6}(101) \rightarrow \vec{U}_{7}(111)$ \\
& $\rightarrow \vec{U}_{6}(101) \rightarrow \vec{U}_{1}(100) \rightarrow \vec{U}_{0}(000)$ \\
\hline
\end{tabular}

The duration time for the two basic active vectors in the first sextant is

$$
\left\{\begin{array}{l}
T_{1}=\frac{\sqrt{3}}{2} M T_{\mathrm{s}} \sin (\pi / 3-\theta) \\
T_{2}=\frac{\sqrt{3}}{2} M T_{\mathrm{s}} \sin \theta
\end{array}\right.
$$

where the modulation index $M$ is always given by

$$
M=\frac{U_{\mathrm{o}}}{U_{\mathrm{DC}} / 2}
$$

The total duration time of the two zero vectors $\left(T_{00}\right.$ for $\vec{U}_{0}$ and $T_{07}$ for $\vec{U}_{7}$ ) is

$$
\begin{aligned}
T_{0} & =T_{\mathrm{s}}-T_{1}-T_{2}=T_{00}+T_{07} \\
& =T_{\mathrm{s}}\left(1-\frac{\sqrt{3}}{2} M \sin (\pi / 3+\theta)\right) \quad(0 \leq \theta \leq \pi / 3)
\end{aligned}
$$

There are three single factor random SVPWM schemes as follows $[18,19]$.

(1) Random switching frequency SVPWM (RSFSVPWM): the switching frequency or the switching period $T_{\mathrm{s}}$ is randomized in an interval.

(2) Random zero-vector distribution SVPWM (RZDSVPWM): the zero vector duration time distribution ratio between the zero vectors $\vec{U}_{0}$ and $\vec{U}_{7}$ is controlled by a random variable $R_{0}$. The duration time $T_{00}$ for $\vec{U}_{0}$ and $T_{07}$ for $\vec{U}_{7}$ is

$$
\left\{\begin{array}{l}
T_{00}=R_{0} T_{0} \\
T_{07}=\left(1-R_{0}\right) T_{0}
\end{array} \quad\left(0 \leq R_{0} \leq 1\right)\right.
$$


(3) Random pulse position SVPWM (RPPSVPWM): the pulse positions of the three phases are controlled by three independent variables $R_{1}, R_{2}$ and $R_{3}$. The duration time is shown in Figure 3 and expresses as

$$
\left\{\begin{array}{l}
t_{1}=R_{1} T_{00} \\
t_{2}=R_{2} T_{1} \\
t_{3}=R_{3} T_{2} \\
t_{4}=T_{07} \\
t_{5}=\left(1-R_{3}\right) T_{2} \\
t_{6}=\left(1-R_{2}\right) T_{1} \\
t_{7}=\left(1-R_{1}\right) T_{00}
\end{array}\right.
$$

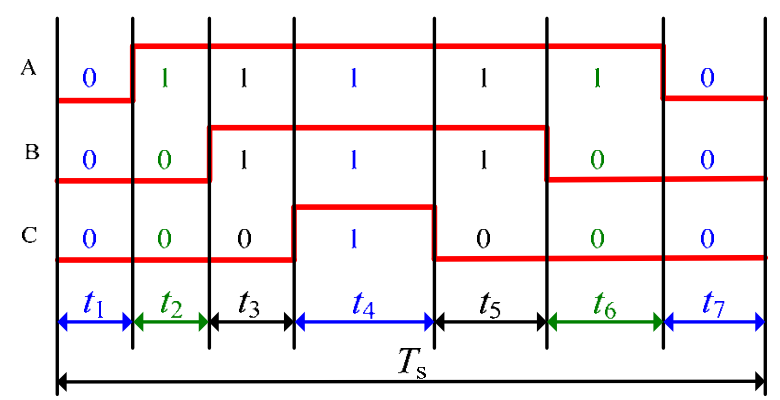

Figure 3. 7-segment SVPWM pattern in the first sextant.

\section{Simulation model}

A simulation model is built in MATLAB/Simulink combined with Plecs based on the control block shown in Figure $4[20,21]$. The proportional-integral (PI) controller is used for the speed controller and current controllers. The 2-Level Converter (Ideal three-phase two-level converter.) block and PMSM (Permanent Magnet Synchronous Machine) block are used directly.

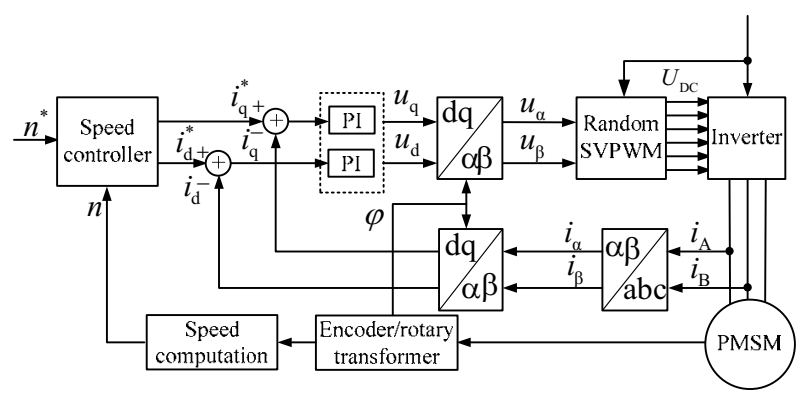

Figure 4. Control block diagram of an electric vehicle.

The random SVPWM subsystem is the key to the simulation and is built using Embedded MATLAB Function block. The flow chart of the random SVPWM subsystem is shown in Figure 5. One random variable is needed in the RZDSVPWM and RSFSVPWM schemes, and three random variables are needed in the RPPSVPWM scheme. The random variable is presented by the random values. The random values that represent the random variables in Equations (5) and (6) should be held constant in the one switching period. Global or persistent variables can be used to store the random values. Persistent variables differ from global variables in that persistent variables are known only to the function in which they are declared. So the persistent variable is more convenient here than the global variable because the random value is only used in one function. The random values are generated at the first simulation step (controlled by the simulation solver) and hold constant during the whole switching period.

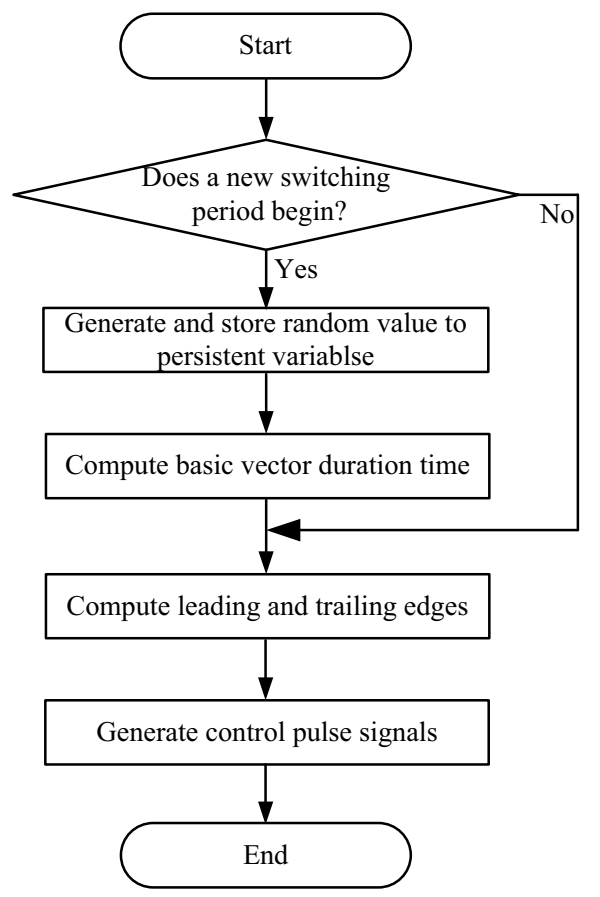

Figure 5. Flow chart of random SVPWM subsystem.

Random functions in MATLAB can be directly used to generate the random values that obey the specified distribution. For example, random (NAME, A, B) or random (NAME, A, B,C) returns an array of random numbers chosen from a two- or three-parameter probability distribution with parameter values $\mathrm{A}, \mathrm{B}$ (and C). $\operatorname{rand}(\mathrm{N})$ returns an $\mathrm{N}-$ by- $\mathrm{N}$ matrix containing pseudorandom values drawn from the standard uniform distribution in the open interval $(0,1)$.

\section{Simulation analysis and discussion}

The simulation experiments are done using the built simulation model described in Section 3. The DC link voltage is $300 \mathrm{~V}$. The number of the pole pairs of PMSM is 4 , the stator resistance is $0.0113 \Omega$, the $\mathrm{d}$-axis and qaxis inductance of the combined stator leakage and magnetizing inductance $[\mathrm{Ld}, \mathrm{Lq}]$ is $[0.000175 \mathrm{H}$, $0.000284 \mathrm{H}$ ], and the flux induced by magnet is 0.08424 $\mathrm{Wb}$. The specified reference speed is $1200 \mathrm{rad} / \mathrm{s}$. All the four random variables in Equations (5) and (6) obey to the standard uniform distribution in the interval $(0,1)$, and the random values are generated using MATLAB function rand. The switching frequency is $15000 \mathrm{~Hz}$ for the deterministic SVPWM scheme, and the interval $(10000 \mathrm{~Hz}, 20000 \mathrm{~Hz})$ with the uniform distribution for 
the RSFSVPWM scheme. The simulation results are shown in Figures 6 to 8.

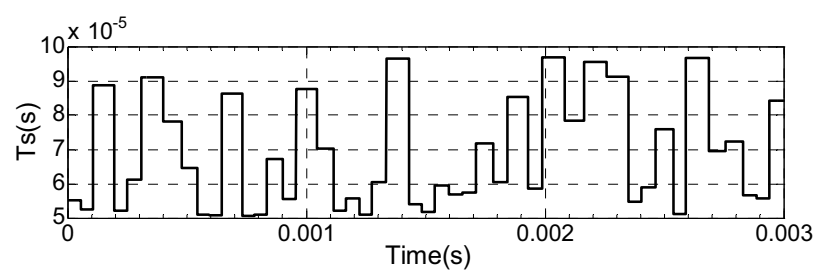

Figure 6. Switching period of RSFSVPWM.

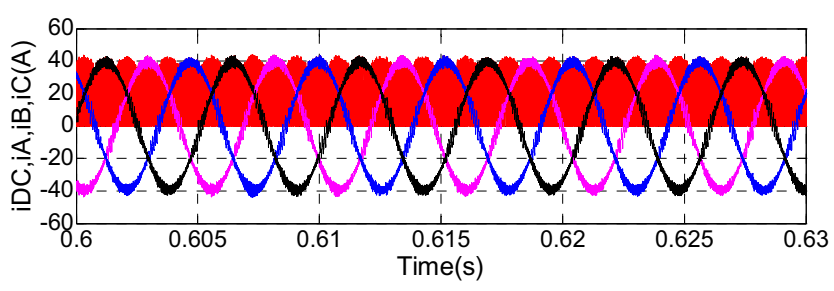

Figure 7. Phases and DC link currents of RSFSVPWM.

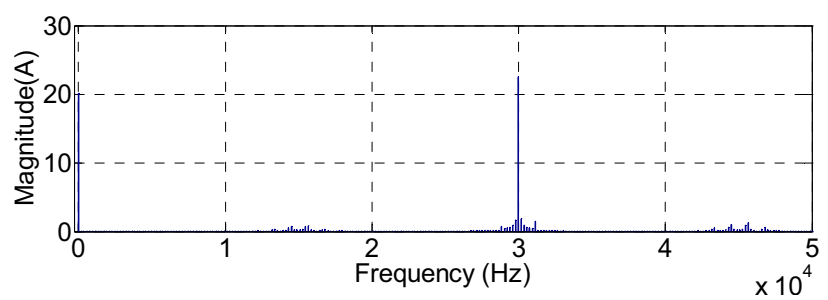

a) Deterministic SVPWM for $15000 \mathrm{~Hz}$.

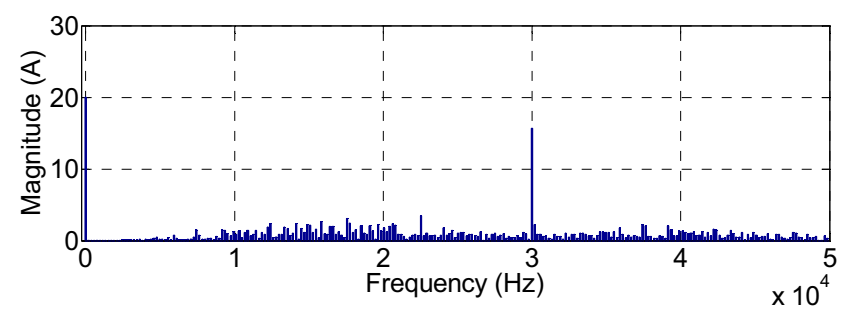

b) RZDSVPWM for $15000 \mathrm{~Hz}$.

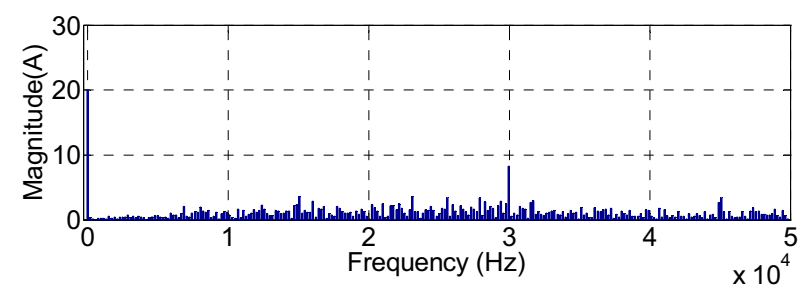

c) RPPSVPWM for $15000 \mathrm{~Hz}$.

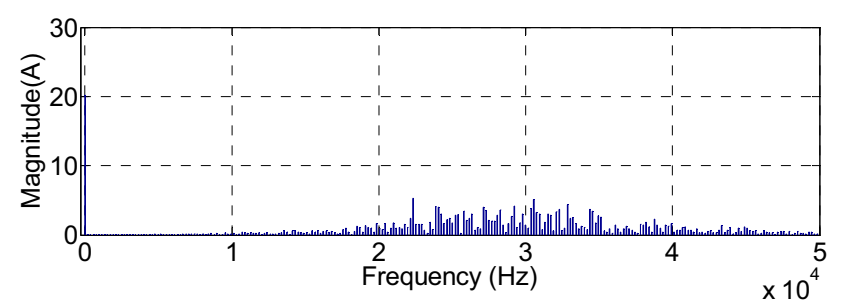

b) RSFSVPWM for $[15000 \mathrm{~Hz}, 20000 \mathrm{~Hz}]$.

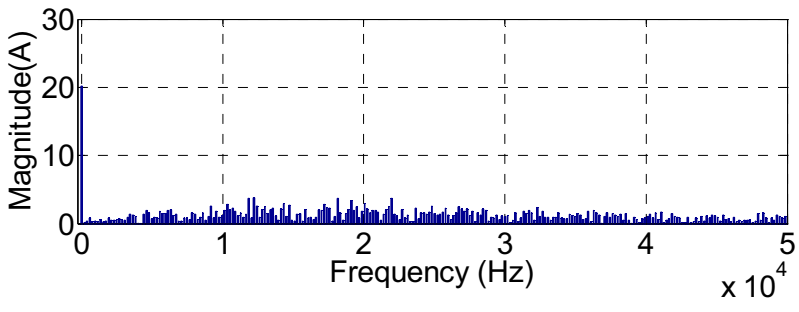

e) Hybrid random SVPWM for [10000Hz, 20000Hz].

Figure 8. Spectra of deterministic and random schemes.

The hybrid SVPWM scheme is a hybrid of not less than two of the three basic random schemes: RZDSVPWM, RPPSVPWM and RSFPWM schemes. The result shown in Figure $8(\mathrm{e})$ is corresponding to the hybrid of the above three random schemes.

The specified reference speed $1200 \mathrm{rad} / \mathrm{s}$ is corresponding to the period $\pi / 600 \mathrm{~s}$. In order to be compared intuitively and conveniently, the DC link currents of the random schemes are sampled in a whole period $\pi / 600 \mathrm{~s}$. The discrete Fourier transform (DFT) function FFT in MATLAB is used to compute the spectra. It should be noticed that there is a little difference between the results in different sampling periods.

Figure 6 presents that the switching period is constantly changing with the time. The switching functions of the three phases are $S_{\mathrm{A}}, S_{\mathrm{B}}$ and $S_{\mathrm{C}}$ that are shown in Figures 2 and 3, and the three phase currents are $i_{\mathrm{A}}, i_{\mathrm{B}}$ and $i_{\mathrm{C}}$. Figure 7 demonstrates that the DC-link current $i_{\mathrm{DC}}$ is the sum of three phase currents and expressed as $i_{\mathrm{A}} S_{\mathrm{A}}+i_{\mathrm{B}} S_{\mathrm{B}}+i_{\mathrm{C}} S_{\mathrm{C}}$ [10]. The randomization makes the pulse leading and trailing edges randomized to some extent, so the switching function and the three phase currents are also randomized to the same extent. Therefore the sum of the three phase currents is also randomized. The randomization can efficiently suppress the peak harmonics of the DC link current. From Figure 8 , we can find that the magnitudes of the harmonics around $30000 \mathrm{~Hz}$ are more than $20 \mathrm{~A}$ for the deterministic SVPWM scheme, but they are about $15 \mathrm{~A}$ for the RZDSVPWM scheme, and less than 10A for the RPPSVPWM, RSFSVPWM and hybrid SVPWM schemes. Especially for the hybrid SVPWM scheme, the magnitudes of the harmonics are less than $5 \mathrm{~A}$.

\section{Conclusions}

A simulation model for the DC link current analysis has been built in MATLAB and presented in this paper. The random SVPWM subsystem is the key to the simulation. The random SVPWM subsystem is built based on the Embedded Function block. In order to keep the random values those represent the variables constant in one switching period, the persistent variables are used. The results presented in the paper show that the random SVPWM scheme can efficiently suppress the harmonic peaks of the DC link current, especially the random 
switching frequency SVPWM scheme has most excellent performance. The simulation method is very flexible in analyzing the DC link current characteristic.

\section{Acknowledgments}

This work is supported by National Science Foundation of China (No. U1304525) and the Science and Technology Key Project of Education Department of Henan Province of China (No. 13B460027). The author would like to thank the anonymous reviewers for their valuable work.

\section{References}

1. F. Wu, J. Zhao, IEEE Trans. Power Electron. 31, 1425 (2016)

2. N. Mutoh, M. Nakanishi, M. Kanesaki, J. Nakashima, IEEE Trans. Electromagn. Compat. 47, 930(2005)

3. H. Shim, H. Kim, Y. Kwack, M. Moon, H. Lee, J. Song, J. Kim, B. Kim, E. Kim, Proc. IEEE Int. Symposium on Electromagn. Compat. (2015)

4. H. Saidi, R. Taleb, N. Mansour, A. Midoun, Proc. IEEE 6th Int. Renewable Energy Congress, 1(2015)

5. G. Zheng, S. Guo, S. Cai, S. Fan, Y. Ren, Proc. IEEE 7th Int. Power Electronics and Motion Control Conf. 4, 2489(2012)

6. N. Rouhana, N. Patin, G. Friedrich, E. Negre, S. Loudot, 17th European Conf. on Power Electronics and Applications, 1(2015)

7. J.W. Kolar, S.D. Round, IEEE Proc.-Electr. Power Appl. 153, 535 (2006)
8. T. D. Nguyen, N. Patin, G. Friedrich, Proc. IEEE Vehicle Power and Propulsion Conf., 1(2011)

9. J. Hobraiche, J. P.Vilain, C. Plasse, IEEE 35th Annual Power Electron. Specialists Conf. 5, 3336 (2004)

10. X. Pei, W. Zhou, Y. Kang, IEEE Trans. Power Electron. 30, 5401(2015)

11. X. Mao, J. A. Kumar, A. Rajapandian, IEEE Trans. Power Electron. 26, 1954 (2011)

12. D. G. Holmes, T. A. Lipo, Pulse Width Modulation for Power Converters: Principles and Practice. (IEEE Press, USA, 2003)

13. K. Shahriyar, M. Javad, A. Ali, IEEE Trans. Ind. Electron. 54, 2333(2007)

14. S. H. Na, Y. G. Jung, Y. C. Lim , S.H. Yang, IEEE Proc.-Electr. Power Appl.149, 195(2002)

15. H.W. Wang, J. Zhang, T. F. Yang, Electron. Measurement Technol. 34, 18(2011)

16. L. Zheng, H. Q. Long, Chinese J. Automotive Eng. 4, 319(2014)

17. M. L Du, Z. M. Xu, L. X. Ding, Automotive Eng. 36,734(2014)

18. G. Q. Chen, Z.H. Wu, Y. Zhu, J. Tongji University 40, 1111(2012).

19. G. Q. Chen, M. J. Zhang, J. W. Zhao, Adv. Inf. Sci. Serv. Sci. 4, 242(2012)

20. A. Kiselev, A. Kuznietsov, R. Leidhold, Proc. 9th Int. Conf. on Compat. and Power Electron., 566 (2015)

21. K. Roumani, B. Schmuelling, Proc. IEEE Conf. on Energy Conversion, 201(2014) 